\title{
Structure of the elastic free energy for chiral nematic liquid crystals
}

\author{
L. Longa \\ Jagellonian University, Department of Statistical Physics, Reymonta 4, Kraków, Poland \\ H.-R. Trebin \\ Universität Stuttgart, Institut für Theoretische und Angewandte Physik, Pfaffenwaldring 57, \\ D-7000 Stuttgart 80, Federal Republic of Germany
}

(Received 6 July 1988)

\begin{abstract}
In Landau-de Gennes theory, the free energy $f$ of liquid crystals is expanded into powers of a symmetric, traceless tensor order parameter $Q_{\alpha \beta}$ and its derivatives $Q_{\alpha \beta, \gamma}$. The expansion is subject to the condition that $f$ is a scalar, i.e., invariant under all rotations of the group $\mathrm{SO}(3)$. Using the method of integrity basis, we have established the most general SO(3)-invariant free-energy density up to all powers in $Q_{\alpha \beta}$ and up to second order in $Q_{\alpha \beta, \gamma}$. It turns out that this free-energy density is composed of 39 invariants, which are multiplied by arbitrary polynomials in $\operatorname{Tr} \underline{Q}^{2}$ and $\operatorname{Tr} \underline{Q}^{3}$. On the other hand, these 39 invariants can be expressed as polynomials of 33 so-called irreducible invariants. Interestingly, among the irreducible invariants there are only three chiral terms (i.e., linear in $Q_{\alpha \beta, \gamma}$ ). They locally give rise to three independent helix modes in chiral, biaxial liquid crystals. This conclusion generalizes results of Trebin [J. Phys. (Paris) 42, 1573 (1981)] and Govers and Vertogen [Phys. Rev. A 31, 1957 (1985); 34, 2520 (1986)] and contradicts a statement of Pleiner and Brand [Phys. Rev. A 24, 2777 (1981); 34, 2528 (1986)], according to which only one twist term is supposed to exist. Some possibilities, leading to a smaller number of the irreducible invariants, are also discussed in detail. These special forms of the elastic free-energy expansion are obtained by imposing additional symmetry restrictions on the field $\underline{Q}$. One arrives, for example, at a system of "soft biaxial nematic" phases by requiring $\operatorname{Tr} Q^{2}=$ const, at a system of biaxial phases with $\operatorname{Tr} Q^{3}=$ const, or at a system with fixed moduli of the order parameter ("hard biaxial" phases) by requiring both. In all cases the number of irreducible invariants is reduced significantly. For example, hard biaxial phases can be described using only 12 irreducible invariants among which three are chiral. Furthermore, three irreducible invariants can be eliminated if surface terms vanish. In all limiting cases relations between irreducible invariants are given explicitly.
\end{abstract}

\section{INTRODUCTION}

It is widely accepted that the orientational properties of liquid crystals can be described by a second-order, symmetric, and traceless tensor $\underline{Q}(\mathbf{r})$ of Cartesian components $Q_{\alpha \beta}(\mathbf{r})(\alpha, \beta=x, y, z)$, where in the standard parametrization

$$
Q_{\alpha \beta}=S\left(n_{\alpha} n_{\beta}-\frac{1}{3} \delta_{\alpha \beta}\right)+P\left(m_{\alpha} m_{\beta}-\frac{1}{3} \delta_{\alpha \beta}\right) .
$$

Here $\hat{\mathbf{n}}, \hat{\mathbf{m}}$, and $\hat{\mathbf{n}} \times \widehat{\mathbf{m}}$ are orthonormal eigenvectors of $\underline{Q}$ corresponding to the eigenvalues $\frac{2}{3} S-\frac{1}{3} P,-\frac{1}{3} S+\frac{2}{3} P$, and $-\frac{1}{3} S-\frac{1}{3} P$, respectively.

By definition the tensor vanishes in the isotropic phase $(S=P=0)$ and thus serves as an order parameter. In the uniaxial nematic, smectic- $A$, etc., ordered phases, $Q(\mathbf{r})$ has uniaxial symmetry, i.e., $S \neq 0, P=0$ or $S=0, P \neq 0$ or $S=P$, where uniaxiality conditions may be written in the equivalent form as one condition

$$
6\left(\operatorname{Tr} \underline{Q}^{3}\right)^{2}=\left(\operatorname{Tr} \underline{Q}^{2}\right)^{3} .
$$

The symmetry axis of the uniaxial phase (director) is defined by the eigenvector of $Q(\mathbf{r})$ corresponding to the nondegenerate eigenvalue. In the most general case $\underline{Q}(\mathbf{r})$ has five independent components. This situation de- scribes the so-called general biaxial phase (GBP). ${ }^{1,2}$

A spatial dependence of $Q$ requires elastic terms, i.e., those which are proportional to derivatives of $Q$ in the Landau free-energy expansion. Together with the thermal contribution the elastic terms form the orientational part of the free energy of liquid crystals.

de Gennes ${ }^{1}$ was the first to propose a Landau-Ginzburg type of expansion for the free-energy density in terms of the tensor order parameter $\underline{Q}$ and its derivatives $Q_{\alpha \beta, \gamma}$. In the absence of electric and magnetic fields, the original expression for the free energy reads

$$
\begin{aligned}
F= & F_{0}+a\left(T-T^{*}\right) \operatorname{Tr} \underline{Q}^{2}+B \operatorname{Tr} \underline{Q}^{3}+C\left(\operatorname{Tr} \underline{Q}^{2}\right)^{2} \\
& +L_{1}^{(2)} Q_{\alpha \beta, \gamma} Q_{\alpha \beta, \gamma}+L_{2}^{(2)} Q_{\alpha \beta, \beta} Q_{\alpha \gamma, \gamma} \\
& +L_{4}^{(2)} \epsilon_{\alpha \beta \gamma} Q_{\alpha \rho} Q_{\beta \rho, \gamma},
\end{aligned}
$$

where the parameters $a, B, C, L_{i}^{(2)}(i=1,2,4)$ are assumed to be independent of temperature $T$. Here $\epsilon_{\alpha \beta \gamma}$ denotes the Levi-Cività tensor. Also summation over repeated indices is to be understood if not stated otherwise. The last term in Eq. (1c) violates parity and is responsible for the formation of a helical ground state. It appears in the elastic free energies of cholesteric liquid crystals.

A further second-order term in $Q_{r \beta . v}$ 


$$
L_{3}^{(2)} Q_{\alpha \beta, \gamma} Q_{\alpha \gamma, \beta}
$$

was omitted by de Gennes because it can be written as linear combination of a surface term and the elastic terms already included in the expansion (1c).

The expansion (1c), though already complicated, is still too simple to describe correctly elastic and thermodynamic properties of real nematic and cholesteric liquid-crystal materials. ${ }^{2-7}$ A possible way out of this difficulty is to include in the expansion (1c) higher-order terms like $Q \underline{Q} \cdots \underline{Q}(\partial \underline{Q})$ and $Q \underline{Q} \cdots \underline{Q}(\partial \underline{Q})(\partial \underline{Q})$. These symbols denote a class of all linearly independent $\mathrm{SO}(3)$-symmetric invariants built up from the tensors $Q_{\alpha \beta} Q_{\gamma \delta} \cdots Q_{\rho \sigma}\left(Q_{\mu \nu, \eta}\right) \quad$ and $\quad Q_{\alpha \beta} Q_{\gamma \delta} \cdots Q_{\rho \sigma}\left(Q_{\mu v, \eta}\right)$ $\times\left(Q_{\xi \tau, \xi}\right)$ by means of contractions with the Kronecker deltas and the Levi-Cività tensors.

Such a program has only partially been carried out as yet. For example, Schiele and Trimper ${ }^{8}$ have found some third-order invariants for uniaxial nematic phases. Their analysis has further been generalized by Berreman and Meiboom $^{9}$ and Poniewierski and Sluckin. ${ }^{10}$ The theories ${ }^{8,9}$ take into account only third-order terms $Q(\partial Q)(\partial Q)$ and apply to strictly uniaxial liquid crystals. In the presence of biaxial fluctuations the general thirdorder theory in $Q_{\alpha \beta}$ becomes unstable and thus is thermodynamically incorrect. One has to include higherorder terms (or neglect third-order ones) to preserve stability of the free energy.

Another drawback of the theories ${ }^{8,10}$ is that they overestimate the number of independent invariants in the general biaxial case and the number of relations between invariants in some limiting cases. The authors use Cartesian tensors. But the Cartesian representation gives only an upper limit for the number of independent terms because the constraints

$$
Q_{\alpha \alpha}=0, \quad Q_{\alpha \alpha, \beta}=0 \quad(\forall \beta)
$$

cannot be easily incorporated into the Cartesian picture.

In our recent paper ${ }^{7}$ we found the correct number of invariants and surface terms up to the fourth order in $Q$. This has been achieved by using the spherical representation for $Q_{\alpha \beta}$ and $\partial_{\mu} Q_{\alpha \beta}$. The spherical representation takes easily into account restrictions (2). However, in orders higher than 4 in $Q$, the direct use of spherical representation also becomes complicated. In these cases the permutation symmetry has to be taken regard of.

The aim of this paper is to show yet another way of expanding the elastic free energy of liquid crystals using the spherical representation. The method is based on a group theoretical construction called integrity basis ${ }^{11}$ (IB).

Rewriting the free energy in terms of an IB offers the following three important advantages:

(i) The expansion is given immediately to an arbitrary order in $Q$.

(ii) The analytical independence of the various terms is evident.

(iii) A systematic analysis is offered of symmetryinduced variations of $Q$ and of the resulting expansion.
Still more, the IB approach uncovers new internal symmetries of the expansion, not easily detected otherwise. For example, we will prove that locally the GBP can be described using only three chiral terms. It also provides a simpler approach to study cholesteric and blue phases, where irreducible representations of space groups play an important role.

The organization of this paper is as follows. In Sec. II some basic rules for the calculation of IB for liquid crystals along with some final formulas are given. In Sec. III the liquid-crystal IB is used to construct a general elastic free energy of nematic and cholesteric phases. In Sec. IV a discussion of surface terms is given. In Sec. V the Cartesian form of the expansion is discussed. Section VI is devoted to a study of the connection between our general theory and some simpler cases, some of which were already proposed in the literature. The paper is concluded with final remarks in Sec. VII.

\section{SO(3)-SYMMETRIC INTEGRITY BASIS FOR THE LIQUID-CRYSTAL ORDER PARAMETER $\underline{Q}$}

The problem often encountered in constructing a Landau-Ginzburg theory of phase transitions is to find all irreducible tensors with respect to a given discrete or compact Lie group, whose components are polynomials in the components of one or more given order parameters. These tensors are described implicitly by a generating function expressed in terms of an integrity basis, i.e., a finite number of "elementary" tensors (polynomials) in terms of which all may be expressed as stretched products. This very elegant group theoretical method has been developed by Judd et al., ${ }^{11(a)}$ Gaskell et al., ${ }^{11(b)}$ and Bistricky et al. ${ }^{11(\mathrm{c})}$ In particular, these authors gave a recipe how to construct integrity bases for an arbitrary set of order parameters forming an irreducible representation of $\mathrm{SO}(3)$.

To discuss the integrity basis for the symmetric and traceless tensor field $Q$ of a liquid crystal, it is convenient to switch from the Cartesian representation to the spherical one. The latter defines the irreducible representations of $\mathrm{SO}(3)$.

The spherical components of $Q$ form an $L=2$ quadrupole tensor $Q^{(2)}$ with components $Q_{m}^{(2)}(m= \pm 2, \pm 1,0)$, where

$$
\begin{aligned}
& Q_{ \pm 2}^{(2)}=-\frac{1}{2}\left(Q_{x x}-Q_{y y} \pm 2 i Q_{x y}\right), \\
& Q_{ \pm 1}^{(2)}= \pm\left(Q_{x z} \pm i Q_{y z}\right), \\
& Q_{0}^{(2)}=\frac{3}{\sqrt{6}}\left(Q_{x x}+Q_{y y}\right) .
\end{aligned}
$$

The spherical components of the vector operator $\partial_{i} \equiv \partial / \partial x_{i}$ form an $L=1$ dipole tensor $\partial_{m}^{(1)}(m= \pm 1,0)$, where

$$
\begin{aligned}
& \partial_{ \pm 1}^{(1)}= \pm \frac{i}{2}\left(\partial_{x} \pm i \partial_{y}\right), \\
& \partial_{0}^{(1)}=i \partial_{z} .
\end{aligned}
$$

In the spherical representation all components $Q_{m}^{(2)}$ are independent, and the constraints $Q_{\alpha \beta}=Q_{\beta \alpha}, \operatorname{Tr} \underline{Q}=0$ of 
the Cartesian representation are already incorporated.

From the components $T_{m}^{(l)}$ and $S_{q}^{(k)}$ of two spherical tensors of rank $l$ and $q$, respectively, one can form $(2 l+1)(2 k+1)$ products $T_{m}^{(l)} S_{q}^{(k)}$, which are reducible. By forming linear combinations weighted by ClebschGordan coefficients, one obtains $(2 l+1)(2 k+1)$ irreducible components transforming according to the angular quantum number $L \in l+k, l+k-1, \ldots,|l-k|$ :

$$
\left[T^{(l)} \otimes S^{(k)}\right]_{m}^{(L)}:=\sum_{m_{1}, m_{2}}\left(\begin{array}{cc|c}
l & k & L \\
m_{1} & m_{2} & m
\end{array}\right) T_{m_{1}}^{(l)} S_{m_{2}}^{(k)},
$$

where

$$
\left[\begin{array}{cc|c}
l & k & L \\
m_{1} & m_{2} & m
\end{array}\right]
$$

are Clebsch-Gordan coefficients. To the product $\left[T^{(l)} \otimes S^{(k)}\right]^{(L)}$ one can couple a third tensor $R_{s}^{(p)}$ to form a spherical product $\left[\left[T^{(l)} \otimes S^{(k)}\right]^{(L)} \otimes R^{(p)}\right]^{(K)}$.

A problem that arises now is that of determining all irreducible $\mathrm{SO}(3)$-symmetric tensors (IT), whose components are homogeneous polynomials in $Q_{m}^{(2)}$. Next, classifying components of derivatives $\partial \underline{Q}$ and $(\partial \underline{Q})(\partial \underline{Q})$ according to the irreducible representations of $\mathrm{SO}(3)$ and by coupling them with IT one can form all (analytically) independent invariants. These invariants appear in the free-energy density expansion.

As already indicated above, to find the explicit algebraic form of the IT, an essential step is the determination of their integrity basis. ${ }^{11}$

The information about IT and IB is easily inferred from a generating function $G(q, \Lambda)$ (Ref. 11) for $\mathrm{SO}(3)$ tensors contained in the symmetric product of an arbitrary number of identical SO(3) tensors.

The generating function is a rational expression whose numerator and denominator are polynomials in $q$ and $\Lambda$. When expanded into a power series in all variables it contains only terms with positive integer coefficients. The power-series expansion of $G(q, \Lambda)$

$$
G(q, \Lambda)=\sum_{n, L} m_{n, L} q^{n} \Lambda^{L}
$$

yields the number of linearly independent IT of degree $n$ and momentum $L$ as the expansion coefficient $m_{n, L}$. Further information about IB is contained in a specific, rational form of $G(q, \Lambda)$ (see example below).

A construction of $G(q, \Lambda)$ is based on the orthogonality theorems for characters of symmetric products of irreducible representations of $\mathrm{SO}(3)$ and has recently been a matter of extensive studies. ${ }^{11}$ In particular, for $Q_{m}^{(2)}$ the result is ${ }^{11(b)}$

$$
\begin{aligned}
G(q, \Lambda)= & \frac{1+q^{3} \Lambda^{3}}{\left(1-q \Lambda^{2}\right)\left(1-q^{2} \Lambda^{2}\right)\left(1-q^{2}\right)\left(1-q^{3}\right)} \\
= & 1+q \Lambda^{2}+q^{2}\left(\Lambda^{0}+\Lambda^{2}+\Lambda^{4}\right) \\
& +q^{3}\left(\Lambda^{0}+\Lambda^{2}+\Lambda^{3}+\Lambda^{4}+\Lambda^{6}\right)+\ldots, \quad|\Lambda|<1 .
\end{aligned}
$$

The rational form of the generating function (5a) may be interpreted in terms of five "elementary" tensors $I_{N}^{(L)}$ whose degrees $N$ and momenta $L$ are, respectively, $(N, L)=(1,2),(2,2),(2,0),(3,0),(3,3)$. These tensors, forming the IB, can uniquely be identified as

$$
\begin{aligned}
I_{1}^{(2)} & \equiv Q^{(2)}, \\
I_{2}^{(2)} & =\left[Q^{(2)} \otimes Q^{(2)}\right]^{(2)}, \\
I_{2}^{(0)} & \equiv I_{2}=\left[Q^{(2)} \otimes Q^{(2)}\right]^{(0)}, \\
I_{3}^{(0)} & \equiv I_{3}=\left[I_{1}^{(2)} \otimes I_{2}^{(2)}\right]^{(0)},
\end{aligned}
$$

and

$$
\begin{aligned}
I_{3}^{(3)} & =\left[I_{1}^{(2)} \otimes I_{2}^{(2)}\right]^{(3)} \\
& =-\frac{\sqrt{5}}{\sqrt{2}}\left[Q^{(2)} \otimes\left[Q^{(2)} \otimes Q^{(2)}\right]^{(4)}\right]^{(3)} .
\end{aligned}
$$

Now, using tensors (6a) and (6b) we can construct an infinite set of tensors of the form

$$
\begin{aligned}
& \left(I_{2}\right)^{m}\left(I_{3}\right)^{n}\left[\left[\bigotimes_{i=1}^{n_{1}} I_{1}^{(2)}\right]^{\left(2 n_{1}\right)} \otimes\left[\bigotimes_{j=1}^{n_{2}} I_{2}^{(2)}\right]^{\left(2 n_{2}\right)}\right]^{\left(2 n_{1}+2 n_{2}\right)}, \\
& \left(I_{2}\right)^{m}\left(I_{3}\right)^{n}\left[I_{3}^{(3)} \otimes\left[\left[\bigotimes_{i=1}^{n_{1}} I_{1}^{(2)}\right]^{\left(2 n_{1}\right)} \otimes\left[\bigotimes_{j=1}^{n_{2}} I_{2}^{(2)}\right]^{\left(2 n_{2}\right)}\right]^{\left(2 n_{1}+2 n_{2}\right)}\right]^{\left(2 n_{1}+2 n_{2}+3\right)},
\end{aligned}
$$

where $m, n, n_{1}$, and $n_{2}$ run over the non-negative integers and where

$$
\begin{aligned}
{\left[\bigotimes_{i=1}^{n} A^{(J)}\right]^{(n J)} } & \equiv\left[\cdots\left[A^{(J)} \otimes\left[A^{(J)} \otimes A^{(J)}\right]^{(2 J)}\right]^{(3 J)} \cdots\right]^{(n J)} .
\end{aligned}
$$

Note that the set of the tensors $(6 \mathrm{c})$ and $(6 \mathrm{~d})$ corresponds formally to the generating function ( $5 a)$ and $(5 b)$

$$
\begin{aligned}
\left(1+\Lambda^{3} q^{3}\right) \sum_{m, n, n_{1}, n_{2}} & \left(q^{2}\right)^{m}\left(q^{3}\right)^{n}\left(q \Lambda^{2}\right)^{n_{1}}\left(q^{2} \Lambda^{2}\right)^{n_{2}} \\
& =\frac{1+q^{3} \Lambda^{3}}{\left(1-q \Lambda^{2}\right)\left(1-q^{2} \Lambda^{2}\right)\left(1-q^{2}\right)\left(1-q^{3}\right)} .
\end{aligned}
$$


We conclude that the irreducible tensors $(6 c)$ and (6d) account for all terms in (5a) and (5b) and fully solve the problem of finding all $\mathrm{SO}(3)$-symmetric tensors build up from $Q^{(2)}$

We would like to emphasize that the Clebsch-Gordan couplings $(6 \mathrm{c})$ and $(6 \mathrm{~d})$ count only these representations that correspond to the highest possible total momenta $2 n_{1}+2 n_{2}$ and $2 n_{1}+2 n_{2}+3$, respectively. This means that the order of the Clebsch-Gordan couplings in $(6 \mathrm{c})$ and $(6 \mathrm{~d})$ is irrelevant and a given set $\left(m, n, n_{1}, n_{2}\right)$ corresponds to only one irreducible tensor. Note also that the tensor $I_{3}^{(3)}$, associated with the term $q^{3} \Lambda^{3}$ in the numerator of $(5 \mathrm{a})$, can appear only linearly in $(6 \mathrm{~d})$. The higherorder couplings of this tensor with itself can be expressed as Clebsch-Gordan couplings between integrity basis elements $(6 a)$.

Finally, let us mention that any other tensor formed by means of coupling of some $Q^{(2)}$ can uniquely be expressed as linear combination of the tensors $(6 \mathrm{c})$ and $(6 \mathrm{~d})$. For example,

$$
\left[I_{1}^{(2)} \otimes I_{2}^{(2)}\right]^{(2)}=\frac{2 \sqrt{5}}{7} I_{2} Q^{(2)}
$$

\section{INVARIANT EXPANSION OF THE FREE ENERGY}

In the previous section we listed all linearly independent, irreducible tensors built up from $Q^{(2)}$. Here we apply these results to construct systematically a general free-energy expansion in terms of $Q$ and its derivatives $Q_{\alpha \beta, \gamma}$. Only terms that are linear or quadratic in derivatives of $Q$ will be considered, i.e., we shall restrict to the $\mathrm{SO}(3)$-symmetric invariants of the form $Q Q \cdots Q(\partial Q)$ and $\underline{Q} \underline{Q} \cdots \underline{Q}(\partial \underline{Q})(\partial \underline{Q})$ or equivalently, using spherical tensor notation

$$
\left[\left[\bigotimes_{i=1}^{n} Q^{(2)}\right]^{(L)} \otimes \partial Q^{(L)}\right]^{(0)}
$$

and

$$
\left[\left[\bigotimes_{i=1}^{n} Q^{(2)}\right]^{(L)} \otimes\left[\partial Q^{\left(L_{1}\right)} \otimes \partial Q^{\left(L_{2}\right)}\right]^{(L)}\right]^{(0)}
$$

where

$$
\partial Q^{(L)} \equiv\left[\partial^{(1)} \otimes Q^{(2)}\right]^{(L)}, \quad L=1,2,3 .
$$

The structure of the tensor space

$$
\left[\bigotimes_{i=1}^{n} Q^{(2)}\right]^{(L)}
$$

has already been studied in the previous section [see Eqs. $(6 \mathrm{c})$ and $(6 \mathrm{~d})]$. Thus, the problem that remains is to solve the identification of all linearly independent, irreducible representations $\partial Q^{(L)}$ and $\left[\partial Q^{\left(L_{1}\right)} \otimes \partial Q^{\left(L_{2}\right)}\right]^{(L)}$. First of all let us note that we can couple $\partial^{(1)}$ and $Q^{(2)}$ to obtain three independent, irreducible representations with $L=1,2,3$, Eq. (7c). Now studying the second case, Eq. (7b) and observing correctly the permutation symmetry with respect to $\partial Q^{(L)}$, one finds that various irreducible representations $\left(L_{1}, L_{2}, L\right)$ can be classified as given in Table I. Clearly, by combining the above results with those of the previous section one finds that the invariants (7a) and 7(b) can be written in an equivalent form, for chiral terms, linear in $\partial Q$

$$
\left(I_{2}\right)^{n_{1}}\left(I_{3}\right)^{n_{2}} J_{\alpha}, \quad \alpha=1,2,3
$$

and for elastic terms, quadratic in $\partial \underline{Q}$,

$$
\begin{aligned}
& \left(I_{2}\right)^{n_{1}}\left(I_{3}\right)^{n_{2}} J_{\alpha} J_{\beta}, \quad \alpha \leq \beta ; \alpha, \beta=1,2,3 \\
& \left(I_{2}\right)^{n_{1}}\left(I_{3}\right)^{n_{2}} D_{\alpha}, \quad \alpha=1, \ldots, 30
\end{aligned}
$$

where $J_{\alpha}$ and $D_{\alpha}$ (together with $I_{2}, I_{3}$ ) are so-called irreducible invariants, i.e., they cannot be expressed as polynomials of invariants of lower order. We can identify 33 irreducible invariants $J_{\alpha}, D_{\alpha}$. They are as follows.

(i) Chiral, $J$ invariants

$$
\begin{aligned}
& J_{1}=\left[Q^{(2)} \otimes \partial Q^{(2)}\right]^{(0)}, \\
& J_{2}=\left[I_{2}^{(2)} \otimes \partial Q^{(2)}\right]^{(0)}, \\
& J_{3}=\left[I_{3}^{(3)} \otimes \partial Q^{(3)}\right]^{(0)} .
\end{aligned}
$$

(ii) $D$ invariants

$$
\begin{aligned}
& {\left[\left[\bigotimes_{i=1}^{n} Q^{(2)}\right]^{(L)} \otimes\left[\partial Q^{\left(L_{1}\right)} \otimes \partial Q^{\left(L_{2}\right)}\right]^{(L)}\right]^{(0)} \cdot \quad(9 \mathrm{~b})} \\
& \text { For } n=0, D_{1}, D_{2}, D_{3} \\
& \quad\left[\partial Q^{(L)} \otimes \partial Q^{(L)}\right]^{(0)}, L=1,2,3 . \\
& \text { For } n=1, D_{4}, \ldots, D_{9} \\
& \quad\left[Q^{(2)} \otimes\left[\partial Q^{(L)} \otimes \partial Q^{(M)}\right]^{(2)}\right]^{(0)}, L \leq M ; L, M=1,2,3 .
\end{aligned}
$$

For $n=2, D_{10}, \ldots, D_{15}$

$$
\left[I_{2}^{(2)} \otimes\left[\partial Q^{(L)} \otimes \partial Q^{(M)}\right]^{(2)}\right]^{(0)}, \quad L \leq M ; L, M=1,2,3 .
$$

For $n=2, D_{16}, \ldots, D_{18}$

$$
\left[\left[Q^{(2)} \otimes Q^{(2)}\right]^{(4)} \otimes\left[\partial Q^{(L)} \otimes \partial Q^{(3)}\right]^{(4)}\right]^{(0)}, \quad L=1,2,3 .
$$

For $n=3, D_{19}, \ldots, D_{21}$

$$
\left[I_{3}^{(3)} \otimes\left[\partial Q^{(L)} \otimes \partial Q^{(M)}\right]^{(3)}\right]^{(0)}, \quad L<M ; L, M=1,2,3 .
$$

For $n=3, D_{22}, \ldots, D_{24}$

$$
\left[\left[I_{1}^{(2)} \otimes I_{2}^{(2)}\right]^{(4)} \otimes\left[\partial Q^{(L)} \otimes \partial Q^{(3)}\right]^{(4)}\right]^{(0)}, \quad L=1,2,3 .
$$

For $n=3, D_{25}$

$$
\left[\left[Q^{(2)} \otimes Q^{(2)} \otimes Q^{(2)}\right]^{(6)} \otimes\left[\partial Q^{(3)} \otimes \partial Q^{(3)}\right]^{(6)}\right]^{(0)} \text {. }
$$

For $n=4, D_{26}, \ldots, D_{28}$

$$
\left[\left[I_{2}^{(2)} \otimes I_{2}^{(2)}\right]^{(4)} \otimes\left[\partial Q^{(L)} \otimes \partial Q^{(3)}\right]^{(4)}\right]^{(0)}, \quad L=1,2,3 .
$$

For $n=4, D_{29}$

$$
\left[\left[I_{1}^{(2)} \otimes I_{1}^{(2)} \otimes I_{2}^{(2)}\right]^{(6)} \otimes\left[\partial Q^{(3)} \otimes \partial Q^{(3)}\right]^{(6)}\right]^{(0)} .
$$

For $n=5, D_{30}$

$$
\left[\left[I_{1}^{(2)} \otimes I_{2}^{(2)} \otimes I_{2}^{(2)}\right]^{(6)} \otimes\left[\partial Q^{(3)} \otimes \partial Q^{(3)}\right]^{(6)}\right]^{(0)},
$$


TABLE I. Combination of irreducible components $\left[\partial^{(1)} \times \underline{Q}^{(2)}\right]^{(M)}$ of the gradient of $\underline{Q}$ with $M=L_{1}$ and $M=L_{2}$ to irreducible tensors of rank $L$ quadratic in the gradient according to Eq. (7b).

\begin{tabular}{ccccccccc}
\hline \hline$L_{1}$ & $L_{2}$ & & \multicolumn{5}{c}{$L$} \\
\hline 1 & 1 & 0 & & 2 & & & \\
1 & 2 & & 1 & 2 & 3 & & \\
1 & 3 & & & 2 & 3 & 4 & & \\
2 & 2 & 0 & & 2 & & 4 & & \\
2 & 3 & & 1 & 2 & 3 & 4 & 5 & \\
3 & 3 & 0 & & 2 & & 4 & & 6 \\
\hline \hline
\end{tabular}

where for two different $D_{\alpha}, D_{\beta}(\alpha<\beta, n$ fixed) the corresponding $L, M$ indices fulfill the condition $L_{\alpha} \leq L_{\beta}$. If $L_{\alpha}=L_{\beta}$ then $M_{\alpha}<M_{\beta}$ is assumed. Note that not all the couplings between spherical tensors $(6 \mathrm{c})$ and (6d) and derivatives $\left[\partial Q^{(M)} \otimes \partial Q^{(N)}\right]^{(L)}$ to total momentum 0 give irreducible invariants. Six of them can be expressed as polynomials of invariants of lower order. For example,

$$
\begin{aligned}
\sqrt{9}\left[\left[I_{1}^{(2)} \otimes I_{2}^{(2)}\right]^{(4)} \otimes\left[\partial Q^{(2)} \otimes \partial Q^{(2)}\right]^{(4)}\right]^{(0)} & \\
& =-D_{2} I_{3}+5 J_{1} J_{2}-\frac{10}{7} I_{2} D_{7} .
\end{aligned}
$$

In deriving (8) and (9) use has been made of the properties of integrity bases and Wigner $6 j$ symbols. ${ }^{12}$

From the analysis as given it follows that the general free-energy density of cholesteric liquid crystals is composed of $30 \mathrm{D}$ invariants, six $J_{\alpha} J_{\beta}$ invariants, and three $J_{\alpha}$ invariants. These can additionally be multiplied by arbitrary polynomials in $I_{2}$ and $I_{3}$.

\section{SURFACE RELATIONS}

Another problem that can systematically be studied using the integrity basis (6a) and the spherical representation is an identification of so-called surface relations. By definition, surface relations are linear combinations of invariants which can be expressed as full divergence. Thus, if due to suitable boundary conditions surface terms vanish, certain invariants become linearly dependent. This allows for further simplification of the free-energy expansion.

The number of surface relations is found in a systematic way as follows. Suppose that elastic invariants have the form (7b). These invariants can be expressed as linear combinations of divergence terms

$$
\left[\partial^{(1)} \otimes\left[\left[\bigotimes_{i=1}^{n+1} Q^{(2)}\right]^{(L)} \otimes\left[\partial^{(1)} \otimes Q^{(2)}\right]^{(L)}\right]^{(1)}\right]^{(0)}
$$

and some higher-order terms of the form

$$
\left[\left[\bigotimes_{i=1}^{n+1} Q^{(2)}\right]^{(M)} \otimes\left[\partial^{(1)} \otimes \partial^{(1)} \otimes Q^{(2)}\right]^{(M)}\right]^{(0)} .
$$

Now suppose that for a given $n$ in (7b), (10a), and (10b) the number of all linearly independent divergence terms
TABLE II. Number of chiral invariants [Eq. (7a)], elastic terms [Eq. (7b)], and surface relations, if the polynomial weighing the gradient terms is of degree $2 n$.

\begin{tabular}{rccc}
\hline \hline & $\begin{array}{c}\text { Invariants [Eq. (7b)] } \\
\text { Elastic } \\
\text { terms }\end{array}$ & $\begin{array}{c}\text { Surface } \\
\text { relations }\end{array}$ & $\begin{array}{c}\text { Invariants } \\
\text { [Eq. (7a)] }\end{array}$ \\
\hline 0 & 3 & 1 & 0 \\
1 & 6 & 1 & 1 \\
2 & 13 & 2 & 1 \\
3 & 17 & 2 & 2 \\
4 & 25 & 3 & 2 \\
5 & 29 & 3 & 3 \\
6 & 37 & 4 & 3 \\
7 & 41 & 4 & 4 \\
8 & 49 & 5 & 4 \\
9 & 53 & 5 & 5 \\
10 & 61 & 6 & 5 \\
\hline \hline
\end{tabular}

(10a) is $U$, the number of invariants (7b) is $V$ and the number of higher-order invariants (10b) generated by divergence terms is $W(U \leq W+V)$. By performing linear combinations of various divergence terms we obtain new $(U-W)$ full divergence terms which are expressed as linear combinations of invariants and in which higher-order invariants (10b) do not appear. They are precisely the surface relations we are looking for. Thus, the number of surface relations is given by the difference $U-W$.

Again, with the help of the IB the calculations of $U$ and $W$ are straightforward. More precisely, the IB gives the full information about possible values of $L$ and $M$ and their multiplicity in the tensor product

$$
\left[\bigotimes_{i=1}^{n+1} Q^{(2)}\right]^{(L)}
$$

Corresponding to these values tensor fields should couple with components of $\left[\partial^{(1)} \otimes Q^{(2)}\right]^{(L)}, L=1,2,3$ to all possible total momenta equal to $1(10 \mathrm{a})$ or with

$$
\left[\partial^{(1)} \otimes \partial^{(1)} \otimes Q^{(2)}\right]^{(M)}, \quad M=0,1,2,3,4
$$

to total momentum 0 [Eq. (10b)]. The allowed values of $M$ are easily inferred from the decomposition of representation $\partial^{(1)} \otimes \partial^{(1)} \otimes Q^{(2)}$ into a direct sum of irreducible representations

$$
\partial^{(1)} \otimes \partial^{(1)} \otimes Q^{(2)}=D^{(0)} \oplus D^{(1)} \oplus 2 D^{(2)} \oplus D^{(3)} \oplus D^{(4)},
$$

where

$$
D^{(M)}=\left[\partial^{(1)} \otimes \partial^{(1)} \otimes Q^{(2)}\right]^{(M)}
$$

is the irreducible representation corresponding to angular momentum $M$.

Now by counting various cases we find that the number of surface relations of order $n$ is equal to the number of solutions of equations $\left\{2 n_{1}+3 n_{2}=n-i, i=0,1,2\right\}$, where $n_{1}, n_{2}\left(n_{1} \geq 0, n_{2} \geq 0\right)$ are integer numbers. Some special cases for $n=1, \ldots, 10$ are listed in Table II. 


\section{CARTESIAN FORM OF IRREDUCIBLE INVARIANTS}

As has been shown in the previous section the spherical representation readily solves the problem of the number and of the form of liquid-crystal invariants and surface relations. In many calculations, however, it is more convenient to use Cartesian representation as one avoids the calculations of Clebsch-Gordan coefficients and the free energy can be written in the most compact form. In our case it is sufficient to find Cartesian counterparts of the irreducible invariants.

For bulk $\left(I_{2}, I_{3}\right)$ and chiral (9a) irreducible invariants this can be done immediately using the proportionalities

$$
\begin{aligned}
& I_{2} \leftrightarrow \operatorname{Tr} \underline{Q}^{2}, \\
& I_{3} \leftrightarrow \operatorname{Tr} \underline{Q}^{3}, \\
& {\left[Q^{(2)} \otimes \partial Q^{(2)}\right]^{(0)} \leftrightarrow \epsilon_{\alpha \beta \gamma} Q_{\alpha \rho} Q_{\beta \rho, \gamma},} \\
& {\left[I_{2}^{(2)} \otimes \partial Q^{(2)}\right]^{(0)} \leftrightarrow \epsilon_{\alpha \beta \gamma} Q_{\alpha \mu} Q_{\mu \delta} Q_{\gamma \delta, \beta},} \\
& {\left[I_{3}^{(3)} \otimes \partial Q^{(3)}\right]^{(0)} \leftrightarrow \epsilon_{\alpha \mu \nu} Q_{\beta \mu}^{2} Q_{\gamma \nu} Q_{\alpha \beta, \gamma} .}
\end{aligned}
$$

For irreducible invariants (9c) the equivalent Cartesian invariants have been found in Ref. 7 for $n=0,1,2$. These invariants are denoted there as

$$
\begin{aligned}
& {\left[L_{1}^{(2)}\right], \ldots,\left[L_{3}^{(2)}\right],} \\
& {\left[L_{1}^{(3)}\right], \ldots,\left[L_{6}^{(3)}\right],} \\
& {\left[L_{5}^{(4)}\right], \ldots,\left[L_{13}^{(4)}\right] .}
\end{aligned}
$$

Similar analysis could, in principle, be repeated for higher-order irreducible invariants, i.e., with $n=3,4$, and 5 .

\section{SPECIAL FORMS OF THE ELASTIC FREE ENERGIES}

In this section we show how some special cases of the liquid-crystal free energy can be obtained from the general expansion in terms of irreducible invariants. They arise as a result of additional symmetry restrictions imposed on the field $Q$. If, for instance, we assume that the long-wavelength distortions of the liquid-crystal state are due to the variation in space of locally preferred directions $\hat{\mathbf{n}}$ and $\hat{\mathbf{m}}$ ("hard biaxial" phases), ${ }^{13}$ then the scalar order parameters $S$ and $P$ can be regarded as position independent which is equivalent to the condition that $I_{2}=$ const and $I_{3}=$ const. On the other hand, restrictions imposed on integrity basis elements generate some relations between irreducible invariants.

The celebrated Frank elastic free energy ${ }^{14}$ of a uniaxial medium serves as another example. In terms of $I_{2}$ and $I_{3}$ the uniaxiality condition ( $1 \mathrm{~b})$ reads

$$
10\left(I_{2}\right)^{3}=7 \sqrt{5}\left(I_{3}\right)^{2} .
$$

With the conditions for the hard biaxial phase added it again leads to a decrease in the number of irreducible invariants. The particular representation ${ }^{14}$ for the invariants in terms of $\hat{\mathbf{n}}$ is obtained if $Q$ is defined by (1a) with $P=0$.
Below we demonstrate how the number of irreducible invariants decreases for "soft biaxial nematic" phases by requiring $\operatorname{Tr} Q^{2}=$ const, for biaxial phases where $\operatorname{Tr} Q^{3}=$ const is required and for hard biaxial phases by requiring both. We also discuss the model of a general, uniaxial liquid crystal in which only condition (11) is fulfilled. Finally we concentrate on hard uniaxial phases by fixing additionally the values of $I_{2}$ and $I_{3}$.

\section{A. Soft biaxial nematic systems}

The integrity basis element $I_{2}$ is the square of the norm of the order parameter $Q^{(2)}$

$$
I_{2}=\left[Q^{(2)}\right]^{2}=\frac{1}{\sqrt{5}} \operatorname{Tr} \underline{Q}^{2} .
$$

Thus with $\operatorname{Tr} \underline{Q}^{2}=$ const we let $Q^{(2)}$ to take the values out of a four-dimensional sphere in five-dimensional space of $Q_{m}^{(2)}$. As the sphere fixes only the norm of the order parameter, which is the sum of the squared axis lengths, but still allows the individual axis lengths to vary in space, we propose to call a system with this property "soft biaxial nematic."

Moving over the four-sphere introduces relations between irreducible invariants. To see this let us note that the condition $\operatorname{Tr} \underline{Q}^{2}=$ const can be written in an equivalent form using derivatives

$$
\begin{aligned}
\frac{1}{\sqrt{5}} \partial_{m}^{(1)}\left(\operatorname{Tr} \underline{Q}^{2}\right) & =\partial_{m}^{(1)} I_{2} \\
& =\frac{2}{\sqrt{5} \sqrt{3}} \sum_{J=1}^{3} \sqrt{2 J+1}\left[Q^{(2)} \otimes \partial Q^{(J)}\right]_{m}^{(1)} \\
& =0 \quad \forall m
\end{aligned}
$$

Now, the vector condition (12a) is equivalent to the set of scalar conditions

$$
\sum_{J=1}^{3} \sqrt{2 J+1}\left[\left[Q^{(2)} \otimes \partial Q^{(J)}\right]^{(1)} \otimes T^{(1)}\right]^{(0)}=0
$$

where

$$
\begin{aligned}
T^{(1)} & \equiv T_{(n, L, \ldots)}^{(1)} \\
& =\left[\left[\bigotimes_{i=1}^{n} Q^{(2)}\right]^{(L \pm 1)} \otimes \partial Q^{(L)}\right]^{(1)} .
\end{aligned}
$$

In order to obtain all possible relations between irreducible invariants it is enough to express $T^{(1)}$ in terms of the integrity basis. We find that all analytically independent tensors $(12 \mathrm{c})$ take the form

$$
\begin{aligned}
& \left(I_{2}\right)^{m}\left(I_{2}\right)^{n} \partial Q^{(1)}, \\
& \left(I_{2}\right)^{m}\left(I_{3}\right)^{n}\left[I_{\alpha}^{(J)} \otimes \partial Q^{(L)}\right]^{(1)}, \\
& \left(I_{2}\right)^{m}\left(I_{3}^{n}\right)\left[\left[I_{\beta}^{(2)} \otimes I_{\gamma}^{(2)}\right]^{(4)} \otimes \partial Q^{(L)}\right]^{(1)},
\end{aligned}
$$

where $J=2, \quad \alpha=1,2, \quad L=1,2,3 ; \quad J=3, \quad \alpha=3, \quad L=2,3$; $(\beta, \gamma)=(1,1),(2,1),(2,2)$. Note that combining $(12)$ and (13) we find only 12 independent relations between irreducible invariants. They correspond to the case $m=n=0$ in (13) and are given in part A of the Physics 
Auxiliary Publication Service (PAPS) material. ${ }^{15}$ In deriving these relations use has been made of the properties of the integrity basis and Wigner $6 j$ and $9 j$ couplings. ${ }^{12}$

We conclude that to describe soft biaxial systems we need only 21 irreducible invariants out of 33 . The relations ${ }^{15}$ allow us to eliminate 12 irreducible invariants: $D_{6}, D_{16}-D_{18}, D_{22}-D_{25}, D_{27}-D_{30}$, where, as a rule, the invariants with the highest possible indices are eliminated. Thus the free-energy density is composed of 27 invariants (three of them being chiral), which are multiplied by arbitrary polynomials in $I_{2}$ and $I_{3}$.

\section{B. Biaxial systems of $\operatorname{Tr} \underline{Q}^{3}=$ const}

We require now that $\operatorname{Tr} Q^{3}=(\sqrt{5} \sqrt{7} / 2 \sqrt{3}) I_{3}=$ const. Invariant $I_{3}$ is the scalar product of two vectors $I_{1}^{(2)}$ and $I_{2}^{(2)}$ belonging to the IB [Eqs. (6)]. Thus

$$
\begin{aligned}
\left(I_{3}\right)^{2} & =\left(\left[I_{1}^{(2)} \otimes I_{2}^{(2)}\right]^{(0)}\right)^{2} \\
& =\left[\left|I_{1}^{(2)}\right|\left|I_{2}^{(2)}\right| \cos (\phi)\right]^{2}, \\
& =\frac{2 \sqrt{5}}{7}\left(I_{2}\right)^{3} \cos (\phi)^{2} \leq \frac{2 \sqrt{5}}{7}\left(I_{2}\right)^{3},
\end{aligned}
$$

where $\phi$ is the "angle" between vectors $I_{1}^{(2)}$ and $I_{2}^{(2)}$ and where the last inequality becomes an equality for $\phi=0$ or $\pi$ which corresponds to uniaxial case [see Eq. (11)].

The biaxial systems of $\operatorname{Tr} Q^{3}=$ const can be analyzed in exactly the same way as soft biaxial systems. Again we start with differentiating the condition $\operatorname{Tr} \underline{Q}^{3}=$ const

$$
\begin{aligned}
\frac{2 \sqrt{3}}{\sqrt{5} \sqrt{7}} \partial_{m}^{(1)}\left(\operatorname{Tr} \underline{Q}^{3}\right) & =\partial_{m}^{(1)} I_{3} \\
& =\frac{\sqrt{3}}{\sqrt{5}} \sum_{J=1}^{3} \sqrt{2 J+1}\left[I_{2}^{(2)} \otimes \partial Q^{(J)}\right]_{m}^{(1)} \\
& =0 \quad \forall m .
\end{aligned}
$$

Now, using conditions (14b) and $T^{(1)}$ defined by (13), we may write down relations analogous to (12b). Again taking $m=n=0$ we find 12 independent relations between irreducible invariants which are listed in part B of the PAPS material. These relations eliminate 12 invariants: $D_{12}, D_{17}, D_{18}, D_{22}-D_{30}$, and final conclusions are similar to those for soft biaxial nematic systems.

\section{Hard biaxial systems}

The degree of ordering for hard biaxial systems is assumed to be unaffected throughout all the medium, i.e., the scalar order parameters $S$ and $P$ do not depend on position and only the vectors $\hat{\mathbf{n}}$ and $\hat{\mathbf{m}}$ are allowed to vary in space. Rewriting these conditions in a spherical basis we find that equivalently $I_{2}$ and $I_{3}$ must be fixed which means that conditions (12b) and (14b) must be fulfilled. We obtain 24 relations between irreducible invariants given in parts A and B of the PAPS material, but only 21 of them are independent. They eliminate 21 invariants: $D_{3}, D_{6}, D_{8}, D_{9}, D_{12}, D_{14}-D_{18}, D_{20}-D_{30}$. We are left with 12 irreducible invariants where still three among them are chiral. Thus, the elastic free energy of hard biaxial systems is built up from 15 invariants, quadratic in derivatives, and three independent chiral invariants. This result agrees with that of Trebin ${ }^{13(a)}$ and Govers and Vertogen ${ }^{13(d)}$. Additional three irreducible invariants may be eliminated, for example $D_{2}, D_{7}, D_{13}$, if surface relations are fulfilled (see Ref. 7).

\section{General uniaxial systems}

A qualitatively different situation than described above is obtained if we require the system be uniaxial. Now, due to the restriction (11), the inequality (14b) becomes an equality and consequently the vectors $I_{1}^{(2)}$ and $I_{2}^{(2)}$ are either parallel, i.e., $\phi=0$ (oblate uniaxial systems) or antiparallel, i.e., $\phi=\pi$ (prolate uniaxial systems). It means that

$$
I_{2} I_{2}^{(2)}=I_{3} I_{1}^{(2)}
$$

Consequently

$$
I_{3}^{(3)}=\left[I_{1}^{(2)} \otimes I_{2}^{(2)}\right]^{(3)}=\frac{I_{2}}{I_{3}}\left[I^{(2)} \otimes I^{(2)}\right]^{(3)}=0 .
$$

Thus, the number of analytically independent elements of integrity basis is now reduced to $I_{2}$ and $I_{2}^{(2)}$.

Equations (15a) and (15b) give very simple relations between irreducible invariants. Substitution (15a) and (15b) into $(9 a)$ and $(9 b)$ yields

$$
\begin{aligned}
& J_{2}=\Lambda J_{1}, \quad J_{3}=0, \\
& D_{\alpha}=\Lambda D_{\alpha-6} \quad(\alpha=10, \ldots, 15), \\
& D_{\beta}=0 \quad(\beta=19,20,21), \\
& D_{\gamma}=\Lambda D_{\gamma-4}=\Lambda^{2} D_{\gamma-10} \quad(\gamma=26,27,28), \\
& D_{30}=\Lambda D_{29}=\Lambda^{2} D_{25},
\end{aligned}
$$

where $\Lambda=I_{3} / I_{2}$. After this enormous reduction we are left with 14 irreducible invariants,

$$
J_{1}, \quad D_{1}-D_{9}, D_{16}-D_{18}, D_{25} \text {. }
$$

These are still not all independent. More precisely, let us note that the most general solution of the equation (15a) reads

$$
I_{1}^{(2)}=\left[V^{(1)} \otimes V^{(1)}\right]^{(2)},
$$

where $V^{(1)}$ is an arbitrary vector field. The solution (17a) guarantees that irreducible invariants of a general, uniaxial phase may be decomposed in the basis of those of the vector field. Since the IB of the vector field $V^{(1)}$ consist of only two tensor fields $V^{(1)}$ and $V_{2}=\left[V^{(1)} \otimes V^{(1)}\right]^{(0)}$, we immediately find that the corresponding set of irreducible invariants counts 14 tensors among which three are linear in derivatives $\partial V^{(L)}, L=0,1,2$. Out of these only eight are polynomials of even order in the number of the components $V_{m}^{(1)}$. Together with $V_{2}$ they may be used to decompose $D$ invariants (16b) and finally to give additional five relations between (16b). These relations are given in part $\mathrm{C}$ of the PAPS material. We conclude that the complete description of a general, uniaxial phase requires nine irreducible invariants,

$$
J_{1}, \quad D_{1}-D_{8} .
$$




\section{E. Hard uniaxial systems}

Last in our reduction scheme is the free energy density of the uniaxial phase in which, in addition to the condition (15a), $I_{2}$ and $I_{3}$ are position independent. To find out independent irreducible invariants for this case it is enough to combine the results $(\mathrm{C} 1)-(\mathrm{C} 5)$ of part $\mathrm{C}$ of the PAPS material with those of part A. We obtain five additional relations, i.e., (C6)-(C10) between the invariants (17b). These eliminate invariants $D_{3}, D_{5}-D_{8}$.

Summarizing, the elastic free energy of hard uniaxial systems requires only four irreducible invariants: $J_{1}, D_{1}, D_{2}, D_{4}$. This number agrees with that of Frank ${ }^{14}$ while a specific form of the Frank elastic free energy is derived by combining the above results with those of Ref. 7.

The last model concludes our demonstration of how the 33 irreducible invariants and two bulk invariants $I_{2}$ and $I_{3}$ of the general, biaxial phase reduce to four invariants of the Frank elastic free energy.

\section{FINAL REMARKS}

In this paper we studied systematically the structure of the elastic free-energy density of biaxial liquid crystals using the concept of integrity bases. The theory is complete up to the terms linear and quadratic in derivatives of the tensor field $Q$. A very important advantage of the analysis in terms of integrity basis is that it directly refers to the symmetry of the order parameter.

Interestingly, the theory predicts a finite number of elementary distortion modes in general, biaxial systems. These can uniquely be identified with irreducible invariants. Among the allowed distortions there are only three types of chiral ones and this number cannot be reduced for biaxial phases. This result contradicts that of Pleiner and Brand. ${ }^{13(e)}$ They argue that the ground state of a chiral biaxial nematic system must consist of a single helix formation and that the description of such a state involves only one twist term. According to the theory presented here elastic terms with $J_{1}$ or $J_{2}$ give rise to different local structures than $J_{3}$ so a ground state with single helix is impossible if these terms compete.

Some insight into the meaning of various irreducible invariants has been achieved by a systematic analysis in Sec. VI of limiting cases for the elastic free energy of general biaxial phases. These limiting cases have been chosen in such a way as to classify all $D$ and $J$ invariants with respect to the internal symmetries of the tensor order parameter. They may be visualized with the help of

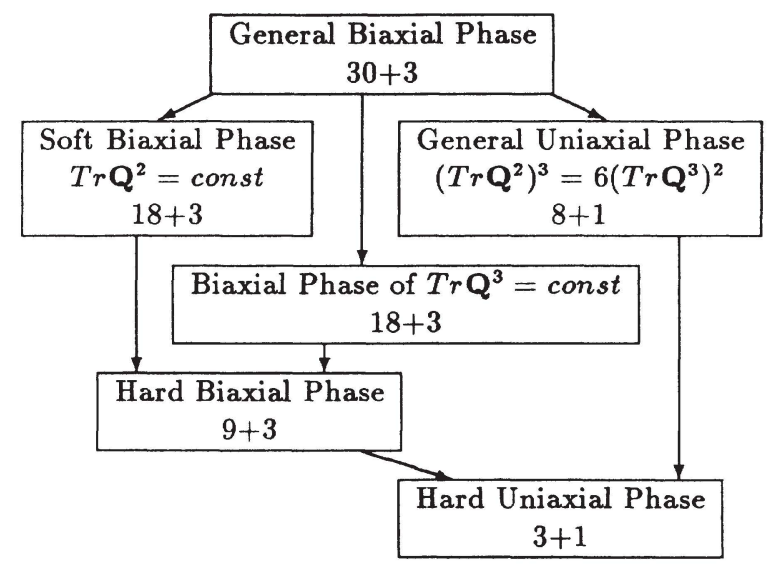

FIG. 1. The different nematic phases which are derived from the general biaxial case by special restrictions. Indicated are the numbers of quadratic plus linear irreducible invariants involved in the construction of the elastic free-energy density for each phase.

Fig. 1, where the numbers of independent irreducible invariants are also given for each case. Of particular importance is the reduction of general uniaxial theory to that governed by uniaxial symmetry. In this case terms proportional to $I_{3}^{(3)}$ vanish and those with $I_{1}^{(2)}$ and $I_{2}^{(2)}$ are simply correlated [Eq. (15a)]. Thus, for uniformly parallel equilibrium structures of nematic phase terms with $I_{3}^{(3)}$ may safely be neglected while between elastic constants of the invariants with $I_{1}^{(2)}$ and $I_{2}^{(2)}$ some simple relations are expected. On the other hand, for strongly twisted equilibrium structures of cholesteric liquid crystals (cholesteric phase, blue phase) the terms governed by the $I_{3}^{(3)}$ element of integrity basis may lead to qualitatively new structures.

Finally we would like to mention that the calculations of the relations between irreducible invariants are tedious by the need to refer explicitly to the values of Wigner 6-j symbols and decompositions of tensor products with respect to the integrity basis. We obtained them using computer algebraic processors MACSYMA and REDUCE.

\section{ACKNOWLEDGMENTS}

This work has been partially supported by the Alexander von Humboldt Foundation and by the Polish Project, Central Project for Fundamental Research (CPBP) No. 01.03 .
${ }^{1}$ P. G. de Gennes, Mol. Cryst. Liq. Cryst. 12, 193 (1971); see also P. G. de Gennes, The Physics of Liquid Crystals (Oxford University Press, 1974).

${ }^{2}$ For a review see, e.g., (a) D. L. Johnson, J. Chim. Phys. 80, 45 (1983); (b) T. C. Lubensky, ibid. 80, 31 (1983).

${ }^{3}$ H. Stegemeyer, Th. Blümel, K. Hiltrop, H. Onusseit, and F. Porsch, Liq. Cryst. 1, 3 (1986).
${ }^{4}$ For a review see, e.g., E. F. Gramsbergen, L. Longa, and W. H. de Jeu, Phys. Rep. 135, 195 (1986).

${ }^{5}$ W. H. de Jeu, Physical Properties of Liquid Crystalline Materials (Gordon and Breach, New York, 1980).

${ }^{6}$ T. C. Lubensky, Phys. Rev. A 2, 2497 (1970).

${ }^{7}$ L. Longa, D. Monselesan, and H.-R. Trebin, Liq. Cryst. 6, 769 (1987). 
${ }^{8}$ K. Schiele and S. Trimper, Phys. Status Solidi B 118, 267 (1983).

${ }^{9}$ D. W. Berreman and S. Meiboom, Phys. Rev. A 30, 1955 (1984).

${ }^{10}$ A. Poniewierski and T. J. Sluckin, Mol. Phys. 55, 1113 (1985)

${ }^{11}$ See, for example, (a) B. R. Judd, W. Miller, Jr., J. Patera, and P. Winternitz, J. Math. Phys. 15, 1787 (1974); (b) R. Gaskell, A. Peccia, and R. T. Sharp, J. Math. Phys. 19, 727 (1978); (c) J. Bystricky, J. Patera, and R. T. Sharp, J. Math. Phys. 23, 1560 (1982)

${ }^{12}$ (a) M. Rotenberg, R. Bivins, N. Metropolis, and J. K. Wooten, The 3-j and 6-j symbols (The Technology Press, MIT, 1959); (b) A. Lindner, Drehimpulse in der Quantenmechanik (Teubner, Stuttgart, 1984).

${ }^{13}$ (a) H.-R. Trebin, J. Phys. (Paris) 42, 1573 (1981); (b) A. Saupe, J. Chem. Phys. 75, 5118 (1981); (c) E. A. Jacobsen and J.
Swift, Mol. Cryst. Liq. Cryst. 78, 311 (1981); (d) E. Govers and G. Vertogen, Phys. Rev. A 31, 1957 (1985); 34, 2520 (1986); (e) H. Pleiner and H. R. Brand, ibid. 24, 2777 (1981); 34, 2528 (1986).

14(a) C. W. Oseen, Trans. Faraday Soc. 29, 883 (1933); (b) H. Zocher, Trans. Faraday Soc. 29, 945 (1933); (c) F. C. Frank, Discussions Faraday Soc. 25, 19 (1958).

${ }^{15}$ See AIP document no. PAPS PLRAA-39-2160-08 for 8 pages of the relations between the irreducible invariants in special cases of the elastic free energy. Order by PAPS number and journal reference from American Institute of Physics, Physics Auxiliary Publication Service, 335 East 45th Street, New York, NY 10017. The price is $\$ 1.50$ for each microfiche $(98$ pages) or $\$ 5.00$ for photocopies of up to 30 pages, and $\$ 0.15$ for each additional page over 30 pages. Airmail additional. Make checks payable to the American Institute of Physics. 